\title{
Attenuation of High-Fat Diet-Induced Rat Liver Oxidative Stress and Steatosis by Combined Hydroxytyrosol- (HT-) Eicosapentaenoic Acid Supplementation Mainly Relies on HT
}

\author{
Francisca Echeverría, ${ }^{1}$ Rodrigo Valenzuela $\left(\mathbb{D},{ }^{1}\right.$ Andrés Bustamante, ${ }^{1}$ Daniela Álvarez, \\ Macarena Ortiz, ${ }^{2}$ Sandra A. Soto-Alarcon, ${ }^{1}$ Patricio Muñoz, ${ }^{1}$ Alicia Corbari, ${ }^{1}$ \\ and Luis A. Videla $\mathbb{D}^{3}$ \\ ${ }^{1}$ Department of Nutrition, Faculty of Medicine, University of Chile, Santiago, Chile \\ ${ }^{2}$ Nutrition and Dietetics School, Faculty of Health Sciences, Catholic University of Maule, Curicó, Chile \\ ${ }^{3}$ Molecular and Clinical Pharmacology Program, Institute of Biomedical Sciences, Faculty of Medicine, University of Chile, \\ Santiago, Chile \\ Correspondence should be addressed to Rodrigo Valenzuela; rvalenzuelab@med.uchile.cl
}

Received 15 January 2018; Revised 18 April 2018; Accepted 26 April 2018; Published 2 July 2018

Academic Editor: Francisco J. Romero

Copyright (c) 2018 Francisca Echeverría et al. This is an open access article distributed under the Creative Commons Attribution License, which permits unrestricted use, distribution, and reproduction in any medium, provided the original work is properly cited.

\begin{abstract}
Pharmacological therapy for nonalcoholic fatty liver disease (NAFLD) is not approved at the present time. For this purpose, the effect of combined eicosapentaenoic acid (EPA; $50 \mathrm{mg} / \mathrm{kg} /$ day) modulating hepatic lipid metabolism and hydroxytyrosol (HT; $5 \mathrm{mg} / \mathrm{kg} /$ day) exerting antioxidant actions was evaluated on hepatic steatosis and oxidative stress induced by a high-fat diet (HFD; $60 \%$ fat, $20 \%$ protein, and $20 \%$ carbohydrates) compared to a control diet (CD; $10 \%$ fat, $20 \%$ protein, and $70 \%$ carbohydrates) in mice fed for 12 weeks. HFD-induced liver steatosis (i) was reduced by $32 \%$ by EPA, without changes in oxidative stress-related parameters and mild recovery of Nrf2 functioning affording antioxidation and (ii) was decreased by $42 \%$ by HT, concomitantly with total regain of the glutathione status diminished by HFD, $42 \%$ to $59 \%$ recovery of lipid peroxidation and protein oxidation enhanced by HFD, and regain of Nrf2 functioning, whereas (iii) combined EPA + HT supplementation elicited $74 \%$ reduction in liver steatosis, with total recovery of the antioxidant potential in a similar manner than HT. It is concluded that combined HT + EPA drastically decreases NAFLD development, an effect that shows additivity in HT and EPA effects that mainly relies on HT, strengthening the impact of oxidative stress as a central mechanism underlying liver steatosis in obesity.
\end{abstract}

\section{Introduction}

Oxidative stress is a disequilibrium condition in which the cellular redox balance is shifted towards a more oxidizing status that may trigger adaptation of cellular functions [1]. Depending on the antioxidant level of different cell types, the concentration of reactive oxygen species (ROS) achieved, and the duration of the exposure, oxidative stress may trigger beneficial responses under mild conditions and potentially harmful ones beneath severe situations, as a typically hormetic phenomenon [2]. In the latter case, oxidative stress development plays a role in the pathogenesis of several liver diseases, including alcoholic liver disease, haemochromatosis,
Wilson's disease, chronic hepatitis $\mathrm{C}$, and nonalcoholic fatty liver disease (NAFLD) [3]. NAFLD is characterized by excess triglyceride (TG) deposition in the hepatocyte followed by development of inflammatory (nonalcoholic steatohepatitis $(\mathrm{NASH})$ ) and fibrogenic responses [4] as shown in patients with obesity and insulin resistance [5-7]. Liver steatosis is also observed in rodents subjected to high-fat diets (HFDs) [8], which are considered adequate experimental models to understand the underlying mechanisms that may support dietary and/or nutritional interventions preventing or treating NAFLD $[9,10]$. In addition to liver steatosis and oxidative stress, HFDs containing $45 \%$ to $75 \%$ of their calories as fat for 12 to 16 weeks induce overweight, insulin resistance, 
a proinflammatory status, apoptosis, and n-3 long-chain polyunsaturated fatty acid (n-3 LCPUFA) depletion [8-11], diets that are characterized by being sufficient in macroand micronutrients $[12,13]$.

Regardless of the high prevalence and increasing incidence of adult and paediatric NAFLD, no pharmacological therapy for NAFLD or NASH is approved at the present time, weight loss and exercise being the mainstay of treatment [14]. Considering that NAFLD is a multifactorial entity, it has been considered that combined therapies may achieve higher rates of responses and improved outcomes than monotherapies [14-16]. Supporting this contention, combined therapies using (i) thyroid hormone- $\left(\mathrm{T}_{3^{-}}\right)$docosahexaenoic acid (DHA) prevent ischemia-reperfusioninduced liver inflammatory injury [17] and (ii) DHA-extra virgin olive oil (EVOO) attenuates HFD-dependent hepatic steatosis [18], whereas (iii) combination drug treatments have been proposed in the case of uncontrolled hypertension [19] and (iv) high-potency statins combined with ezetimibe or pioglitazone were recommended for the resolution of NAFLD or NASH [20]. Based on the hepatoprotective effects of the EVOO component hydroxytyrosol (HT) [21, 22] and the n-3 LCPUFA eicosapentaenoic acid (EPA) [23], we hypothesized that the combined supplementation with EPA and HT could alter HFD-induced biochemical changes associated with steatosis. For this purpose, general metabolic parameters were measured concomitantly with the fatty acid (FA) composition and degree of steatosis, the oxidative stress status, and the activity of antioxidant enzymes controlled by the redox-sensitive transcription factor nuclear factor erythroid 2-related factor 2 (Nrf2) in the liver of mice subjected to $\operatorname{HFD}(60 \%$ of the total calories as fat for 12 weeks).

\section{Methods}

2.1. Animals and Diet Supplementation. Weaning male C57BL/6J mice weighing 12-14g (Bioterio Central, ICBM, Faculty of Medicine, University of Chile) were randomly assigned to each experimental group $(n=8$ per experimental group) and were allowed free access to control diet (CD) or HFD. The CD composition (expressed as \% total calories) was $10 \%$ fat, $20 \%$ protein, and $70 \%$ carbohydrate, with a caloric value of $3.85 \mathrm{kcal} / \mathrm{g}$, and HFD composition was $60 \%$ fat, $20 \%$ protein, and $20 \%$ carbohydrate, with a caloric value of $5.24 \mathrm{kcal} / \mathrm{g}$ (Rodent Diet, product data D12450B and D12492, Research Diet Inc., USA). FA composition of CD and HFD was previously described [9]. Animals received water ad libitum and were housed on a $12 \mathrm{~h}$ light/dark cycle from days 1 to 84 (12 weeks). EPA, isolated from fish oil (Golden Omega S.A., Chile) as TAG (50\% EPA, 5\% DHA, and $5 \%$ of other n-3 FAs; $15 \%$ saturated fatty acid (SFA) (principally palmitic acid), and 25\% MUFA (principally oleic acid)), was administered at $50 \mathrm{mg} / \mathrm{kg} /$ day dosage. HT (ela$\mathrm{Vida}^{\mathrm{TM}}$, DSM Nutritional Products Company, Netherlands) was given at doses of $5 \mathrm{mg} / \mathrm{kg} / \mathrm{day}$, and control groups received isovolumetric amounts of saline orally, conforming eight experimental groups, namely, (a) CD (control), (b) $\mathrm{CD}+\mathrm{EPA}$, (c) $\mathrm{CD}+\mathrm{HT}$, (d) $\mathrm{CD}+\mathrm{EPA}+\mathrm{HT}$, (e) HFD, (f) $\mathrm{HFD}+\mathrm{EPA}$, (g) HFD + HT, and (h) HFD + EPA + HT. The doses of EPA or HT used in this study were used according to previous research; namely, EPA $(50 \mathrm{mg} / \mathrm{kg})$ represents $50 \%$ of the dose of EPA + DHA used by Valenzuela et al. [8], whereas $\mathrm{HT}$ at $5 \mathrm{mg} / \mathrm{kg}$ exhibits protective effects against HFD [24]. Weekly controls of body weight and diet intake were performed through the whole period, and at the end of the 12th week the animals were fasted (6-8 h) and anesthetized with isoflurane (Lunan Baxter Pharmaceuticals Co. Ltd., Shandong, China), and blood samples were obtained by cardiac puncture for the determination of serum aspartate transaminase (AST) and alanine transaminase (ALT), together with the oxidative stress status of the liver. Liver samples were either frozen in liquid nitrogen for determination of FA composition or fixed in phosphate-buffered formalin, embedded in paraffin, stained with haematoxylineosin, and analysed by optical microscopy in a blind fashion describing the presence of steatosis and inflammation, both graded as absent, mild, moderated, and severe [25].

2.2. Ethics. All animal procedures in this study were in strict adherence to the Guide for the Care and Use of Laboratory Animals (National Academy of Sciences, NIH Publication 6-23, revised 1985) and were approved by the Bioethics Committee for Research in Animals, Faculty of Medicine, University of Chile (CBA protocol 0580 FMUCH).

2.3. Biochemical Analyses and Oxidative Stress Markers. Serum AST and ALT activities $\left(\mathrm{UL}^{-1}\right)$ were measured using specific diagnostic kits (bioMérieux SA, Marcy l'Etoile, France). Total fat content in liver $(\mathrm{mg} / \mathrm{g})$ was evaluated according to Bligh and Dyer [26], and triacylglycerol (TAG) content $\left(\mathrm{mgg}^{-1}\right)$ was measured using specific kits according to the manufacturer's instructions (Cayman Chemical Company, Michigan, USA). Livers from anesthetized animals were perfused in situ with a cold solution containing $150 \mathrm{mM} \mathrm{KCl}$ and $5 \mathrm{mM}$ Tris ( $\mathrm{pH} 7.4$ ) to remove blood for protein carbonylation and glutathione assessments. Protein carbonyl concentration was determined by a fluorometric assay (Cayman Chemical Company, Michigan, USA) after adjusting the total protein concentration to $7.5 \mathrm{mg} \mathrm{mL}^{-1}$ per sample. Reduced glutathione (GSH) and glutathione disulphide (GSSG) contents were assessed with an enzymatic recycling method [27]. The antioxidant capacity of serum, serum and liver thiobarbituric acid reactive substances (TBARS), and hepatic F-8 isoprostanes were determined by colorimetric assays (Cayman Chemical Company, Michigan, USA).

2.4. Determination of Liver Antioxidant Enzyme Activities. The liver activity of CAT was measured according to the method of Lück [28]. Assessment of SOD activity was carried out with a commercial assay kit (Cayman Chemical Company; Michigan, USA) according to the manufacturer's instructions. GPX activity was determined using the method described by Paglia and Valentine [29]. GR activity was determined according to Horn [30], NADPH-quinone oxidoreductase 1 (NQO1) activity was measured according to the method of Ernster et al. [31], glutathione-S-transferase (GST) activity was determined according to the method 
described by Habig et al. [32], and $\gamma$-glutamyl transpeptidase (GGT) activity was determined following the method of Satomura et al. [33]. To develop the experimental specific conditions for evaluating the liver activity of these enzymes, we followed the methods previously published by RincónCervera et al. [34] and Valenzuela et al. [24].

2.5. Gene Expression Assays. Total RNA was isolated from liver samples using TRIzol (Invitrogen, Paisley, UK), according to the supplier's protocols. Purified RNA $(2 \mu \mathrm{g})$ was then treated with DNase (DNA-free kit; Ambion, Austin, TX, USA) and used to generate first-strand cDNA with M-MLV Reverse Transcriptase (Invitrogen, Paisley, UK), utilizing random hexamers (Invitrogen, Paisley, UK) and dNTP mix (Bioline, London, UK), according to the manufacturer's protocol. The resultant cDNA was amplified with specific primers for mice in a total volume of $10 \mu \mathrm{L}$. Table 1 depicts the gene-specific primer sequences used in the study. Primer optimization and real-time quantitative PCR were performed according to Rincón-Cervera et al. [34].

2.6. Assessment of Liver DNA-Binding Activity of Nrf2. Nuclear extracts from liver tissue (left lobe) were obtained using a commercial extraction kit (Cayman Chemical Company, Michigan, USA). Nrf2 DNA-binding activity was assessed with commercial ELISA kits (Cayman Chemical Company, Michigan, USA), according to the manufacturer's instructions. Values were expressed as percentage of Nrf2 DNA binding with respect to a positive control provided by the ELISA kit.

2.7. FA Profile. Quantitative extraction of total lipids from liver was carried out according to Bligh and Dyer [26]. Liver samples were homogenized in ice-cold chloroform/ methanol $(2: 1 \mathrm{v} / \mathrm{v})$ containing $0.01 \%$ butylated hydroxytoluene in an Ultra-Turrax homogenizer (Janke \& Kunkel, Stufen, Germany). Total lipids from liver samples were extracted with chloroform/methanol $(2: 1 \mathrm{v} / \mathrm{v})$. Fatty acid methyl esters (FAMEs) from total liver fat were prepared as previously described [34] and analysed according to Valenzuela et al. [9].

2.8. Statistical Analysis. Statistical analysis was performed with GraphPad Prism version 6.1 (GraphPad Software, San Diego, CA, USA). Values shown represent the mean \pm SEM for the number of separate experiments indicated. Two-way ANOVA and Bonferroni's post hoc test assessed the statistical significance of differences between mean values, with $p<0.05$ being considered significant. Pearson's coefficient was used to assess associations between variables.

\section{Results}

3.1. General Parameters, Food and Energy Intake, and Liver Function-Related Indexes. Mice in the different experimental groups exhibiting comparable initial body weights showed $75 \%$ to $95 \%$ increases in their final body weights when given $\mathrm{CD}$, with an average body weight gain of $12.4 \pm 0.7 \mathrm{~g}(n=64)$ that was enhanced by $85 \%$ by HFD alone (group e; Table 2 ). EPA or EPA + HT supplementation in HFD mice generated a significant reduction in body weight gain, $20 \%$ and $33 \%$, respectively, whereas HT supplementation was not significant in this parameter (Table 2). In all experimental groups, dietary intake was comparable, but energy consumption was higher in mice subjected to HFD without or with EPA, $\mathrm{HT}$, and $\mathrm{EPA}+\mathrm{HT}$ supplementation over values in the respective CD groups. Under these conditions, serum AST and ALT levels were comparable in all groups, whereas liver weight in the HFD + EPA + HT group was reduced by $18 \%$ $(p<0.05)$ compared to $\mathrm{CD}$ values. Furthermore, hepatic total fat was comparable in mice subjected to $C D$ without or with supplementations, similarly to liver TG levels; however, hepatic fat increased by $193 \%$ due to HFD, and liver TGs were elevated by $210 \%$ over $C D$ values $(p<0.05)$. The latter two parameters in HFD mice were not altered by EPA or HT, but decreased by $75 \%$ and $63 \%(p<0.05)$ by EPA + HT supplementation, respectively (Table 2 ).

3.2. Liver Morphological Characteristics. Mice subjected to CD without or with EPA, HT, and EPA + HT supplementation exhibited normal liver histology (Figures 1(a)-1(d)) and showed comparable liver steatosis scores measured according to [35] (Figure 1(i)). HFD for 12 weeks elicited macrovesicular liver steatosis (Figure 1(e)) with 6.5-fold elevation in the steatosis score compared to the $\mathrm{CD}$ group $(p<0.05)$, a change that was decreased by $32 \%, 42 \%$, and $74 \%$ by EPA, HT, and EPA + HT supplementation, respectively $(p<0.05)$ (Figure $1(\mathrm{i})$ ).

3.3. Liver FA Composition. Total liver SFA, MUFA, and PUFA levels were comparable in all groups subjected to CD (Table 3). However, (i) total SFAs showed 35\% increase by HFD over CD values $(p<0.05)$, which was decreased by $25 \%, 41 \%$, and $22 \%$ by EPA, HT, and EPA + HT supplementation $(p<0.05)$; (ii) total MUFAs were not modified in all experimental groups; and (iii) total PUFAs were decreased by $35 \%$ by HFD over CD levels $(p<0.05)$, a change that was reduced by $35 \%, 38 \%$, and $38 \%$ by EPA, HT and EPA + HT supplementation, respectively $(p<0.05)$ (Table 3$)$. In relation to total LCPUFAs, mice subjected to CD and EPA or EPA + HT supplementation showed $26 \%$ or $34 \%$ increases over CD alone $(p<0.05)$, whereas HFD-fed animals exhibited $41 \%$ reduction over $\mathrm{CD}$ values, an alteration that was reversed by $58 \%, 38 \%$, and $68 \%$ by EPA, HT, and EPA + HT supplementation, respectively $(p<0.05)$ (Table 3 ). Similarly, HFD induced (i) $35 \%$ decrement in n-6 LCPUFA levels $(p<0.05)$ compared to $\mathrm{CD}$ values, with EPA, HT, and $\mathrm{EPA}+\mathrm{HT}$ eliciting $20 \%, 18 \%$, and $30 \%$ recovery, respectively $(p<0.05)$; (ii) $53 \%$ reduction in n-3 LCPUFAs $(p<0.05)$ over $\mathrm{CD}$ values, whereas EPA, HT, and EPA + HT reached $161 \%, 74 \%$, and $169 \%$ rescue versus $\mathrm{HFD}$ alone, with $115 \%$ and $130 \%$ enhancement by EPA and EPA + HT being found in mice given $\mathrm{CD}$ alone; and (iii) $37 \%$ increase in the n-6/n-3 LCPUFA ratio $(p<0.05)$, which was lowered by $54 \%, 32 \%$, and $51 \%$ by EPA, HT, and EPA + HT $(p<0.05)$, respectively (Table 3 ).

3.4. Blood Plasma and Liver Oxidative Stress-Related Parameters. Mice subjected to CD showed similar values of 
TABLE 1: Gene-specific primer sequences used in the study.

\begin{tabular}{lcc}
\hline mRNA & Forward primer & Reverse primer \\
\hline Nrf2 & AAGCTTTCAACCCGAAGCAC & TTTCCGAGTCACTGAACCCA \\
Gst & TGCAGACCAAAGCCATTCTC & ACGGTTCCTGGTTTGTTCCT \\
Ggt & ATGTGGACACCCGATGCAGTATT & TGTCTTGCTTGTAGTCAGGATGGTT \\
$\beta$ - Actin & ACTGCCGCATCCTCTTCCTC & CTCCTGCTTGCTGATCCACATC \\
\hline
\end{tabular}

Sequences are listed in the $5^{\prime} \rightarrow 3^{\prime}$ direction. Nrf2: nuclear factor erythroid 2-related factor 2; Gst: glutathione-S-transferase; Ggt: $\gamma$-glutamyl transpeptidase.

the antioxidant capacity of plasma when given EPA, which was enhanced by $65 \%$ by HT and EPA $+\mathrm{HT}$ supplementation $(p<0.05)$; however, HFD elicited $58 \%$ reduction in animals without or with EPA treatment that was enhanced by $120 \%$ in HT and EPA + HT groups $(p<0.05)$ (Figure $2(a)$ ). Animals given $\mathrm{CD}$ without and with supplementations exhibited no significant changes in liver total GSH equivalents (Figure 2(b)), in the levels of GSH (Figure 2(c)) and GSSG (Figure 2(d)), in GSH/GSSG ratios (Figure 2(e)), and in the content of TBARS (Figure 2(f)), F-8 isoprostanes (Figure $2(\mathrm{~g})$ ), or protein carbonyls (Figure $2(\mathrm{~h})$ ). HFD led to significant decreases in total GSH equivalents (30\%), GSH content (34\%), and GSH/GSSG ratios (44\%), with no alteration in GSSG levels, whereas the contents of TBARS, F-8 isoprostanes, and protein carbonyls were increased by $154 \%, 157 \%$, and $215 \%$, respectively, over the CD group, changes that were comparable to those found in the HFD + EPA group (Figures 2(b)-2(h)). Compared to the group given HFD alone, HT and EPA + HT recovered to $\mathrm{CD}$ values hepatic total GSH equivalents and GSH/GSSG ratios (Figures 2(b) and 2(e)); furthermore, HT and EPA + HT recovered the contents of liver GSH by $77 \%$ and $84 \%$ (Figure 2(c)), TBARS by $59 \%$ and $68 \%$ (Figure $2(\mathrm{f})$ ), F-8 isoprostane by $42 \%$ and $48 \%$ (Figure $2(\mathrm{~g})$ ), and protein carbonyls by $43 \%$ and $47 \%$ (Figure $2(\mathrm{~h})$ ), respectively. Under these conditions, antioxidant parameters in plasma (antioxidant capacity) and liver (GSH levels) were significantly correlated $(r=0.84 ; p<0.004)$, whereas liver GSH contents were inversely associated with those of TBARS $(r=-0.98 ; p<$ $0.0001)$, F-8 isoprostanes $(r=-0.94 ; p<0.0002)$, and protein carbonyls $(r=-0.93 ; p<0.0004)$.

3.5. Liver Nrf2 DNA Binding; mRNA Expression of Nrf2, GST, and GGT; and Activity of Enzymes Controlled by Nrf2. Mice subjected to CD without and with supplementations revealed comparable values of Nrf2 DNA-binding capacity and in the mRNA expression of Nrf2, GST, and GGT, which were significantly decreased by $68 \%, 77 \%, 76 \%$, and $59 \%$ over CD values by HFD $(p<0.05)$ (Figures 3(a)-3(d)). HFD-induced decrease in Nrf2 DNA binding exhibited 33\% and 64\% recovery by EPA and $\mathrm{HT}$, respectively, whereas EPA $+\mathrm{HT}$ achieved total recovery (Figure 3(a)); similarly, Nrf2 mRNA levels were recovered by $32 \%, 55 \%$, and $93 \%$ by EPA, HT, and EPA $+\mathrm{HT}$, respectively (Figure 3(b)). Compared to the group given HFD alone, GST mRNA levels were recuperated by $22 \%, 25 \%$, and $67 \%$ by EPA, HT, and $\mathrm{EPA}+\mathrm{HT}$, respectively (Figure 3(c)); likewise, EPA, HT, and EPA + HT improved by $8 \%, 48 \%$, and $70 \%$ HFD-induced reduction in GGT mRNA expression (Figure 3(d)).
EPA, HT, and EPA + HT did not elicit significant changes in the activities of the studied antioxidant enzymes in mice fed CD; however, HFD alone decreased those of CAT (66\%), SOD (62\%), GPX (45\%), GR (60\%), NQO1 (66\%), GST (76\%), and GGT $(59 \%)(p<0.05)$ (Figures $4(\mathrm{a})-4(\mathrm{~g}))$. In HFD-treated animals, (i) EPA did not alter GPX, NQO1, GST, and GGT activities (Figures 4(c), 4(e), 4(f), and 4(g)), but it recovered by $54 \%, 30 \%$, and $36 \%$ those of CAT, SOD, and GR, respectively (Figures 4(a), 4(b), and 4(d)), and (ii) HT improved SOD activity by $31 \%$ (Figure $4(\mathrm{~b})$ ), whereas (iii) HT and HT + EPA totally recuperated the activities of CAT, GPX, GR, NQO1, GSR, and GGT (Figures 4(a), 4(c)-4(g)).

3.6. Correlations. Liver steatosis score was significantly correlated with the content of hepatic TAGs $(r=0.94 ; p<0.0002)$ and fat content $(r=0.97 ; p<0.0001)$ and inversely associated with the antioxidant capacity of plasma $(r=-0.75 ; p<0.02)$. The hepatic levels of the antioxidant GSH exhibited an inverse association with the prooxidant parameters, TBARs $(r=-$ $0.98 ; p<0.0001)$, protein carbonyls $(r=-0.92 ; p<0.0004)$, and F-8 isoprostanes $(r=-0.92 ; p<0.0012)$, but revealed a direct correlation with the DNA binding of the redoxsensitive transcription factor Nrf2 $(r=0.94 ; p<0.0004)$. Moreover, the DNA-binding activity of $\mathrm{Nrf} 2$ was significantly associated with the activities of the antioxidant enzymes CAT $(r=0.94 ; p<0.0003)$, SOD $(r=00.95 ; p<0.0001)$, GPX $(r=0.89 ; p<0.002)$, GR $(r=0.92 ; p<0.0005)$, NQO1 $(r=0.89 ; p<0.002)$, GST $(r=0.93 ; p<0.0005)$, and GGT $(\mathrm{r}=0.89 ; p<0.002)$.

\section{Discussion}

Mice subjected to a HFD comprising $60 \%$ of the total calories as fat for 12 weeks developed macrovesicular steatosis as evidenced histologically, with a 6.5 -fold increase in the steatosis score over the $C D$ values, in agreement with previous studies using either the same dietary protocol $[8-10,18,34]$ or alternate procedures [11-13]. Under these conditions, total SFAs were increased by HFD, whereas total PUFAs including $n-6$ and n-3 LCPUFAs were reduced, changes that may contribute to fatty liver development. HFD-induced liver steatosis score was correlated with the significant enhancements in the contents of liver total fat and TGs, changes that were elicited under conditions of a comparable dietary intake but increased energy intake, and negatively associated with oxidative stress development. The latter phenomenon was characterized by significant alterations in the GSH status lowering the antioxidant potential of the liver with the consequent lipid peroxidation and protein oxidation responses, shown 


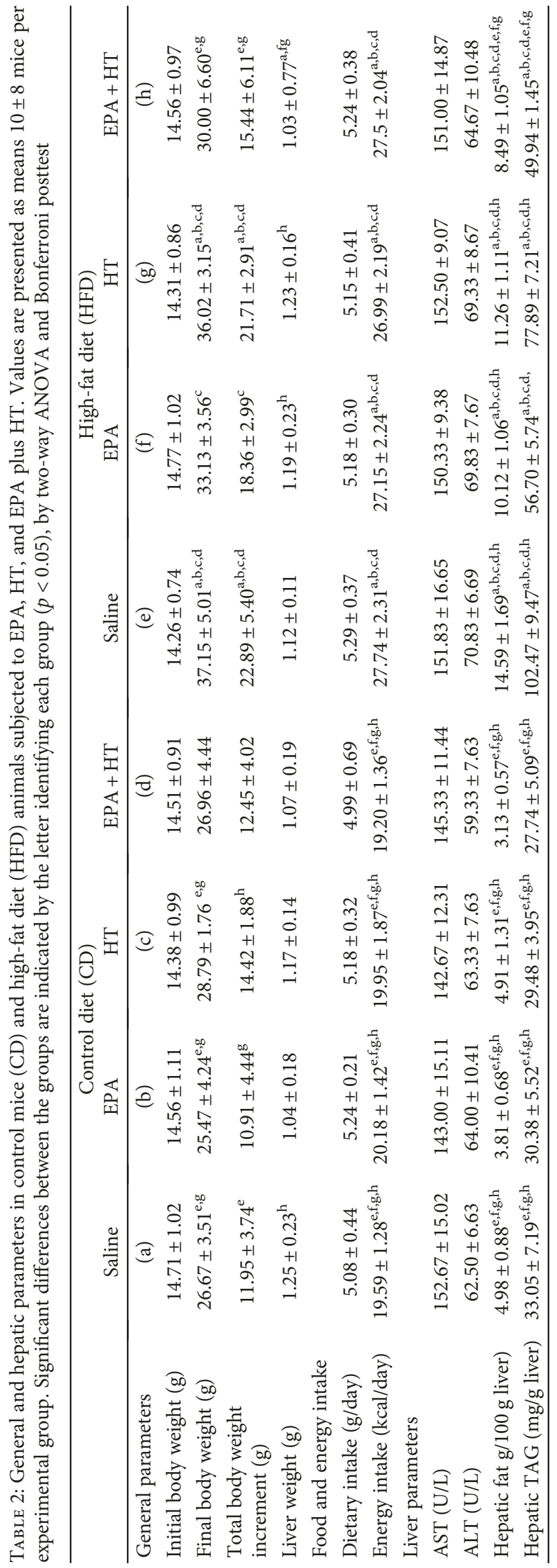


CD

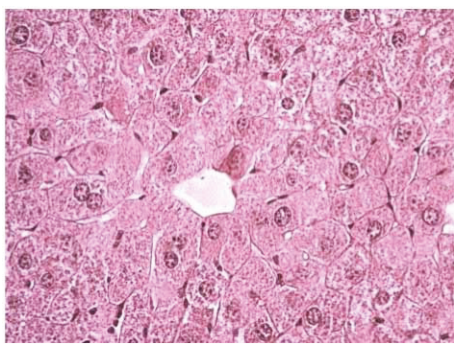

(a)

$\mathrm{CD}+\mathrm{EPA}+\mathrm{HT}$

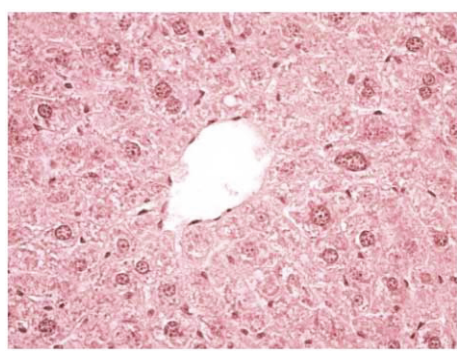

(d)

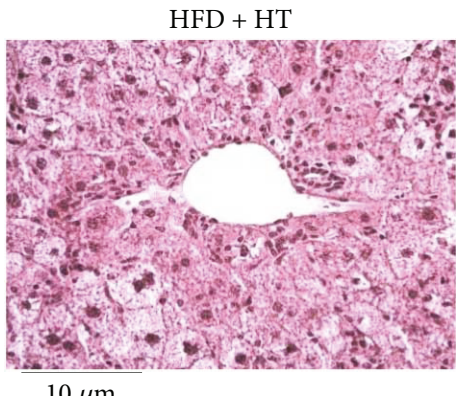

(g)
$\mathrm{CD}+\mathrm{EPA}$

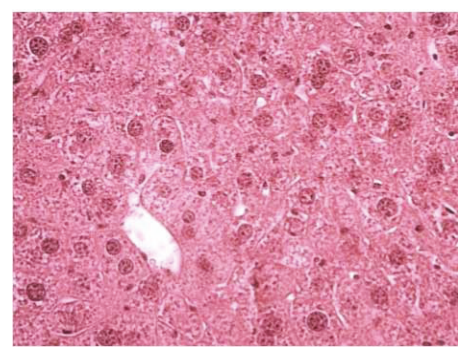

(b)

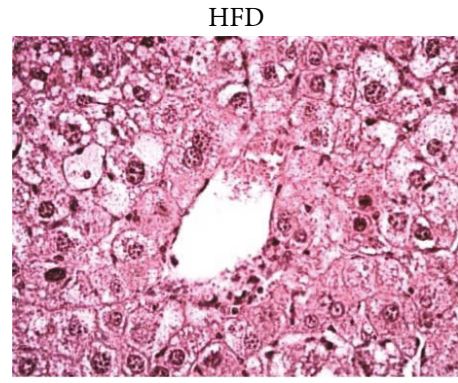

(e)

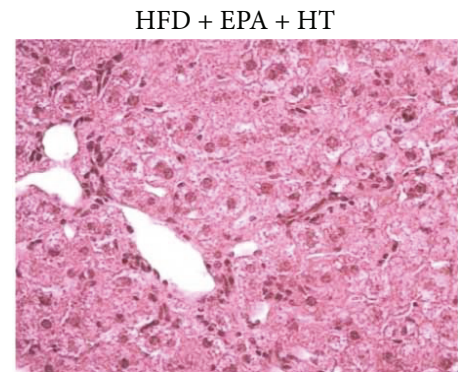

(h)

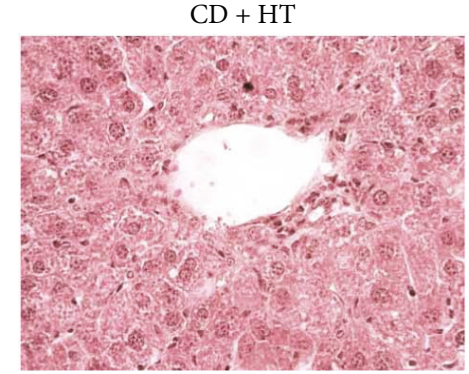

(c)

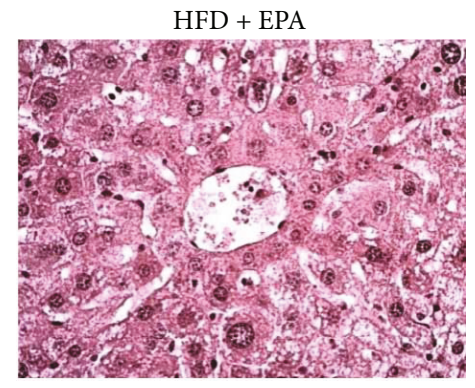

(f)

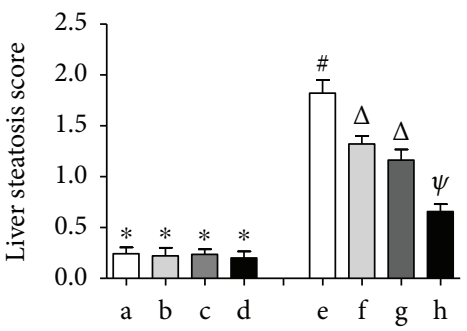

(i)

FIGURE 1: Liver histological assessment in mice subjected to control diet (CD) and high-fat diet (HFD) without and with eicosapentaenoic acid (EPA), hydroxytyrosol (HT), and EPA + HT supplementation. Representative liver sections from animals given (a) CD, (b) CD + EPA, (c) CD + HT, (d) CD + EPA + HT, (e) HFD, (f) HFD + EPA, (g) HFD + HT, and (h) HFD + EPA + HT (haematoxylin-eosin liver sections from 8 animals per experimental group; original magnification $\times 40)$. (i) Liver steatosis scores [24] expressed as means \pm SEM for 8 animals per experimental group. Groups sharing the same symbol are not significantly different among them according to two-way ANOVA and the Bonferroni posttest $(p<0.05) .{ }^{*}, \#, \Delta$ and $\psi$ indicate the significant differences between the experimental groups.

by the elevations in the levels of hepatic TBARs, F-8 isoprostanes, and protein carbonyls, which were inversely correlated with those of GSH. Enhancement of the hepatic oxidative stress status by HFD may be contributed by the drastic decrease in the operation of Nrf2 shown by the lowered Nrf2 DNA-binding capacity and Nrf2 mRNA expression compared to $\mathrm{CD}$, leading to reduced mRNA expression and/or activity of the antioxidant enzymes controlled by Nrf2. Loss of liver Nrf2 activity under sustained oxidative stress conditions triggered by HFD may be related to (i) the prevailing high free-radical level promoting protein oxidation (Figure 2(h)) and inactivation and/or (ii) the increase in the expression of the Nrf2 inhibitor Kelch-like ECHassociated protein 1 (Keap1) which supports continuous proteasomal Nrf2 degradation [36], a mechanism that remains to be evaluated after HFD feeding.
EPA is one of the most important n-3 LCPUFAs due to its roles as (i) DHA precursor, (ii) regulator of hepatic lipid metabolism, which is accomplished by activation of peroxisome proliferator-activated receptor $\alpha$ (PPAR- $\alpha$ ) favouring FA oxidation and downregulation of sterol regulatory element-binding protein 1c (SREBP-1c) reducing de novo lipogenesis [23], and (iii) inhibitor of nuclear factor- $\kappa \mathrm{B}$ (NF- $\kappa \mathrm{B})$ limiting inflammatory processes, actions that are shared by DHA [37]. At the dosage of $50 \mathrm{mg} / \mathrm{kg} /$ day, EPA supplementation significantly increased the hepatic content of EPA as well as that of DHA, both in mice subjected to CD or HFD. Under these conditions, however, HFDinduced liver steatosis was reduced by only $32 \%$ by EPA, without alterations in oxidative stress-related parameters and mild recovery of Nrf2 functioning. This is probably related to transformations that EPA could undergo in the 


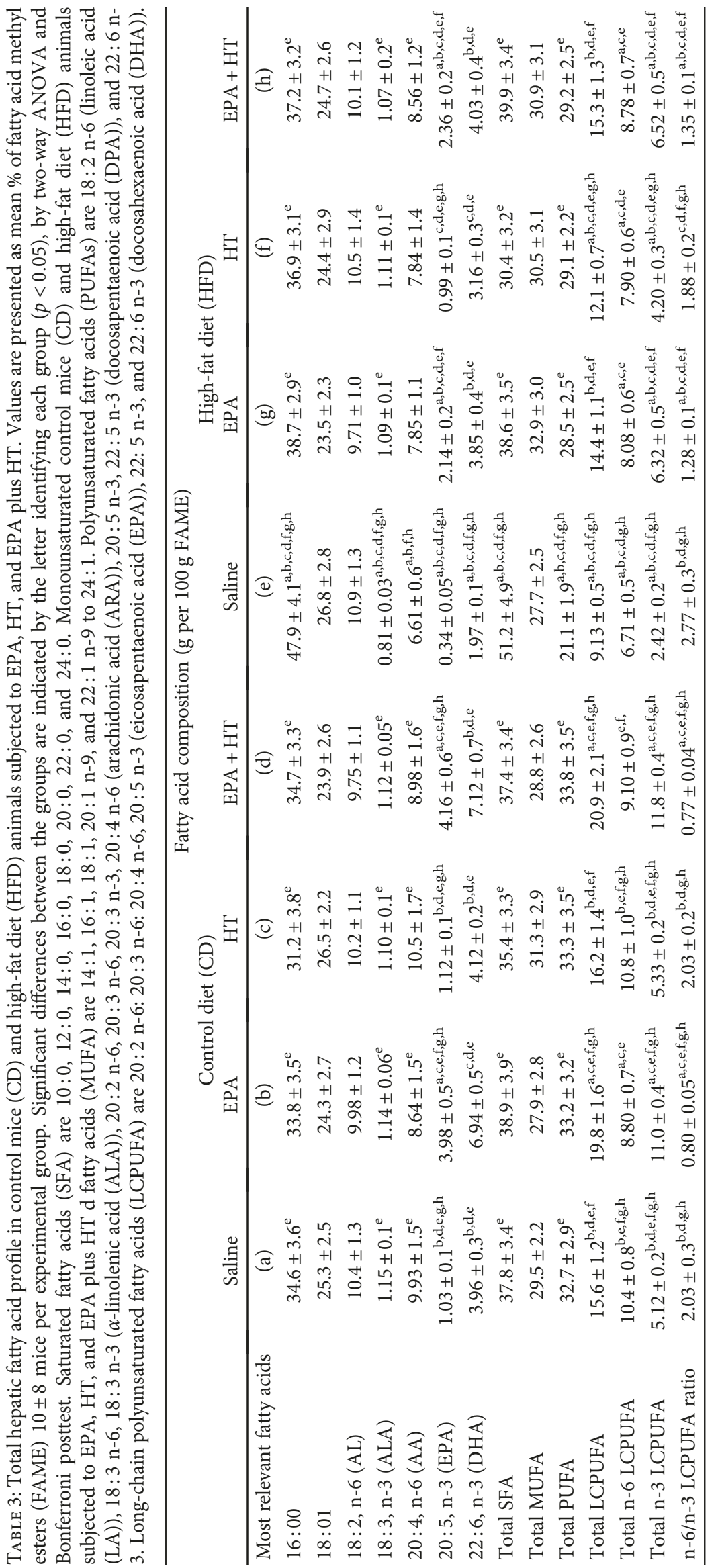




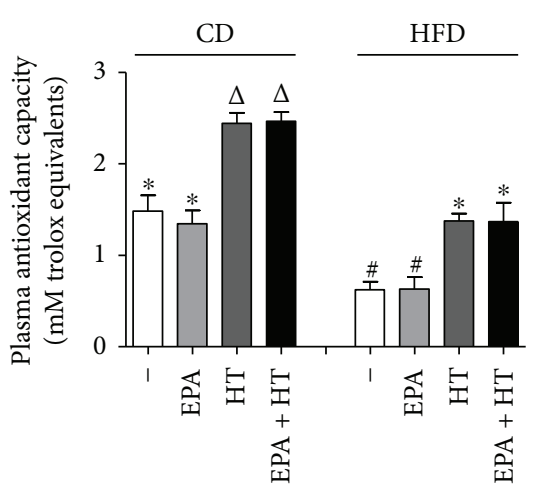

(a)

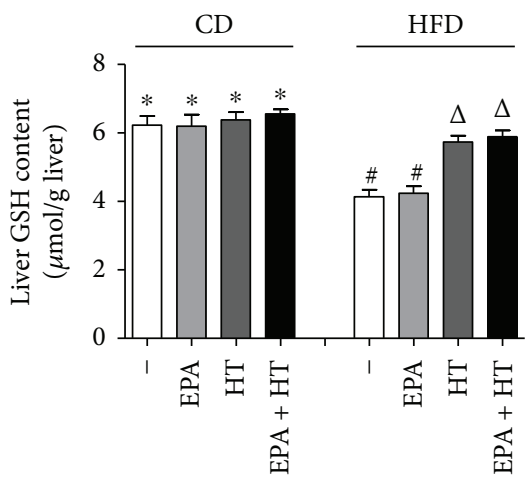

(c)

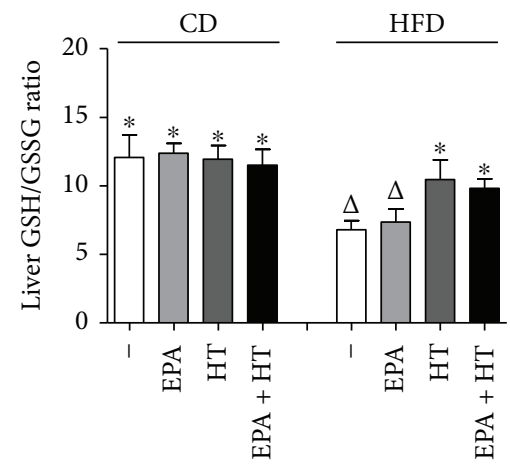

(e)

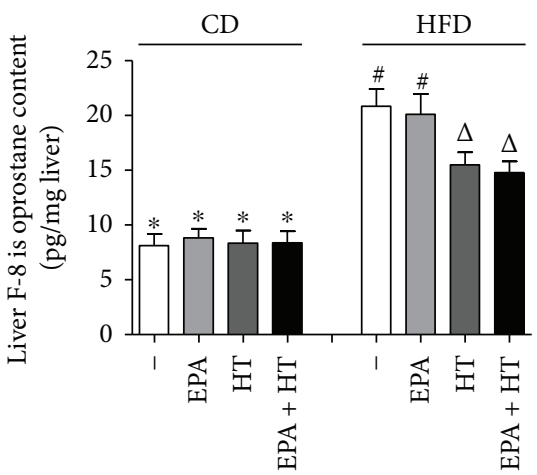

(g)

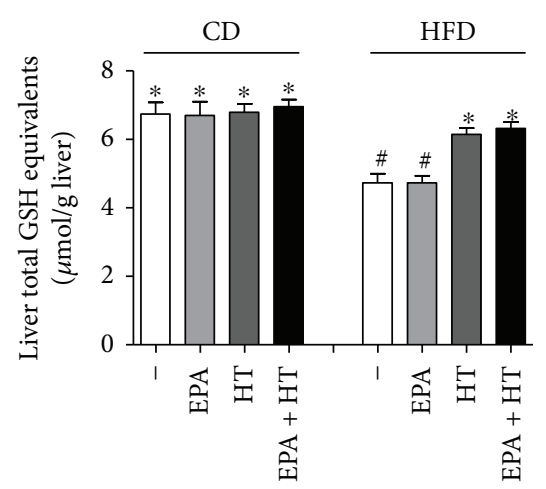

(b)

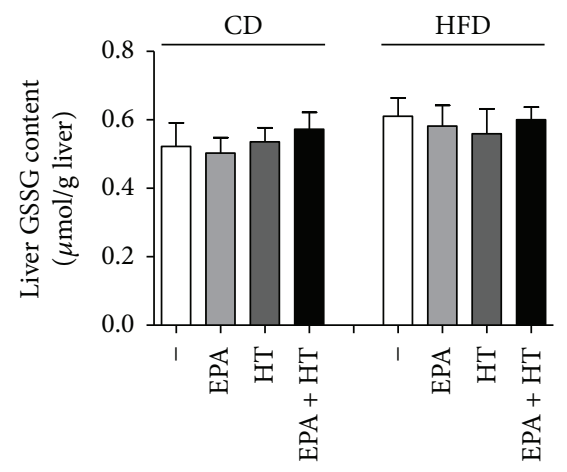

(d)

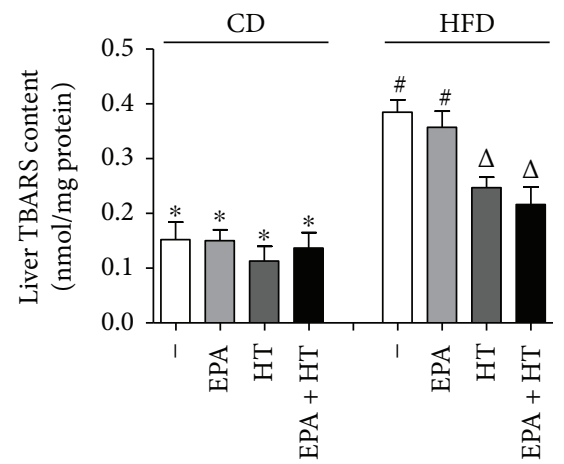

(f)

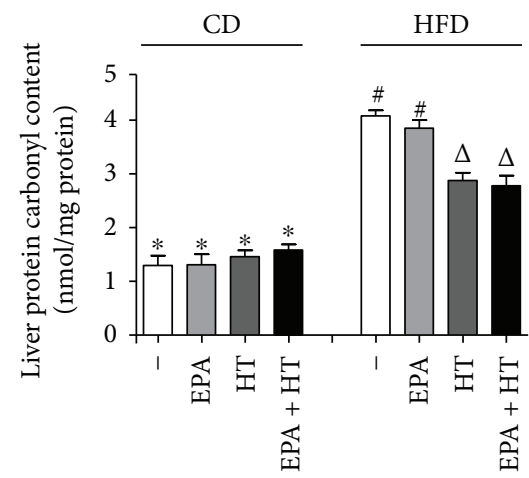

(h)

FIGURE 2: Liver oxidative stress-related parameters in mice subjected to control diet (CD) and high-fat diet (HFD) without (-) and with eicosapentaenoic acid (EPA), hydroxytyrosol (HT), and EPA + HT supplementation. Antioxidant capacity of plasma (a) and contents of total GSH equivalents (b), GSH (c), GSSG (d), GSH/GSSG ratios (e), TBARs (f), F-8 isoprostanes (g), and protein carbonyls (h). Values are means \pm SEM for 8 animals per experimental group. Groups sharing the same symbol are not significantly different among them according to two-way ANOVA and the Bonferroni posttest $(p<0.05)$. GSH: reduced glutathione; GSSG: glutathione disulphide; TBARs: thiobarbituric acid reactants. ${ }^{*}, \#$ and ${ }^{\Delta}$ indicate the significant differences between the experimental groups. 


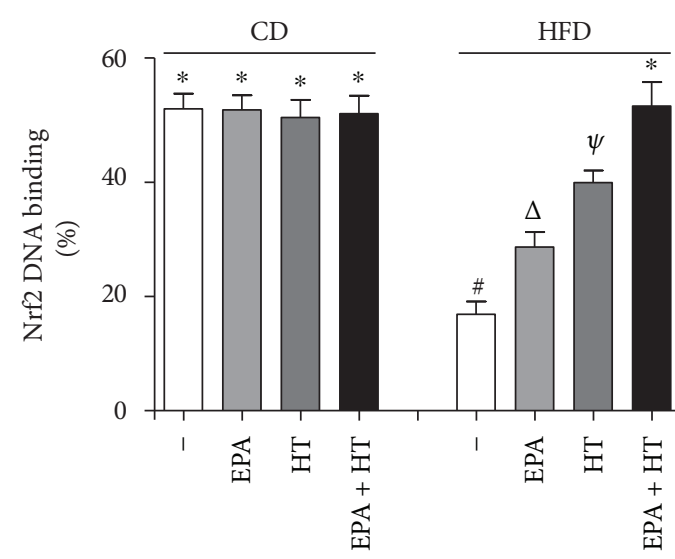

(a)

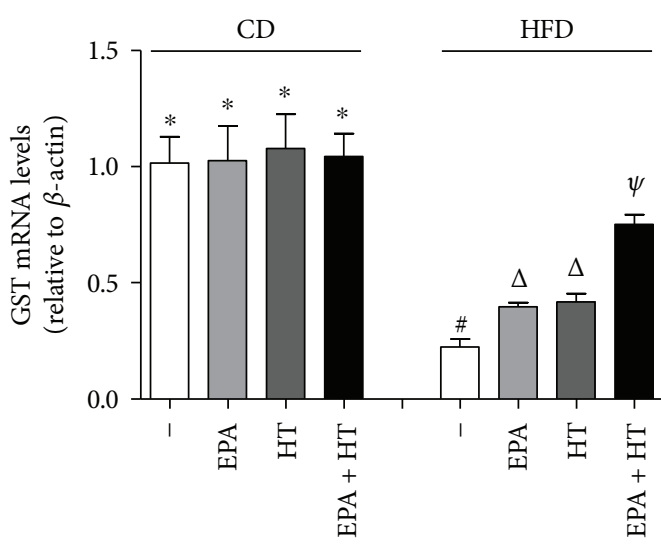

(c)

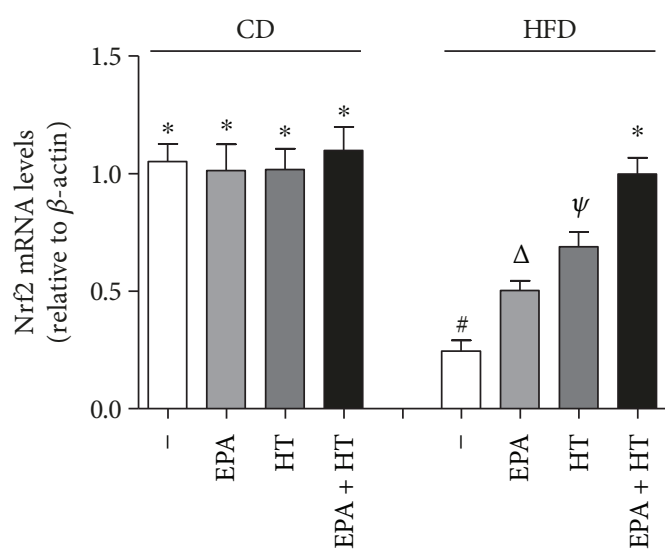

(b)

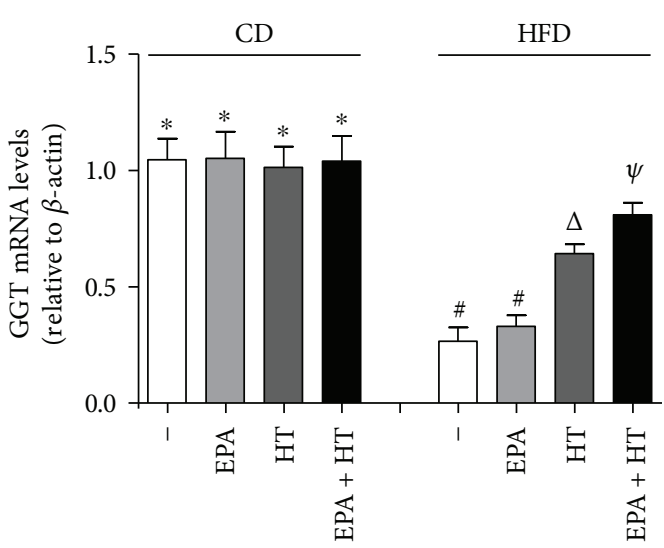

(d)

FIGURE 3: Liver Nrf2 DNA binding (a) and mRNA expression of Nrf2 (b), GST (c), and GGT (d) in mice subjected to control diet (CD) and high-fat diet (HFD) without (-) and with eicosapentaenoic acid (EPA), hydroxytyrosol (HT), and EPA + HT supplementation. Values are means \pm SEM for 8 animals per experimental group. Groups sharing the same symbol are not significantly different among them according to two-way ANOVA and the Bonferroni posttest $(p<0.05)$. Nrf2: nuclear factor erythroid 2-related factor 2; GST: glutathioneS-transferase; GGT: $\gamma$-glutamyl transpeptidase. ${ }^{*, \#, \Delta}$ and $\psi$ indicate the significant differences between the experimental groups.

liver, producing metabolic products such as DHA, E-resolvins [38], epoxy-derivatives [39], and/or $\mathrm{J}_{3}$ isoprostanes [40], thus limiting the concentration needed for cell signaling.

HT is a polyphenol present in EVOO that has a powerful antioxidant action [41] regulating different signaling pathways associated with the intracellular redox state [42]. This is in agreement with the significant increases in the antioxidant capacity of plasma by HT observed in CD and HFD groups, an effect that is correlated with (i) the total regain towards $\mathrm{CD}$ values of the glutathione status (total GSH equivalents and GSH/GSSG ratio), with $77 \%$ recovery of GSH levels that were decreased by HFD, and (ii) the partial recovery ( $42 \%$ to $59 \%$ ) of parameters related to free radicalinduced lipid peroxidation (TBARs and F-8 isoprostanes) and protein carbonylation that were enhanced by HFD, indexes negatively correlated with GSH levels. Under these conditions, reduction in HFD-induced liver oxidative stress status by HT is associated with enhancement $(55 \%)$ in the mRNA expression of Nrf2, with $64 \%$ regain in its DNAbinding capacity, responses that may contribute to an increase in the antioxidant potential of the liver [24]. This contention is supported by the total recovery of the activity of the enzymes controlled by Nrf2 that were decreased by HFD, namely, CAT, GPX, GR, NQO1, GST, and GGT, with the partial regain in that of SOD observed after HT supplementation. An additional effect elicited by HT favouring the antioxidant potential of the liver under HFD-induced oxidative stress conditions is the enhancement in the content of total LCPUFAs including that of total n-3 LCPUFAs, possibly by decreasing their oxidative deterioration [43]. This effect of HT is likely to promote PPAR- $\alpha$ activation increasing the FA oxidation capacity of the liver with concomitant SREBP-1c downregulation reducing de novo lipogenesis [43], thus in agreement with the $42 \%$ reduction found in HFD-induced steatosis score that correlated with the decreases in the fat and TG contents.

HFD-fed mice subjected to combined supplementation with $\mathrm{EPA}+\mathrm{HT}$ showed a significant greater and additive antisteatotic effect ( $74 \%$ reduction) compared to that elicited by the separate HT (42\% diminution) and EPA (32\% decrease) treatments, with hepatic fat and TG contents being significantly lower than those induced by HFD alone. However, the effects of EPA $+\mathrm{HT}$ along with HFD feeding were either (i) similar to those achieved by HT alone, namely, total 


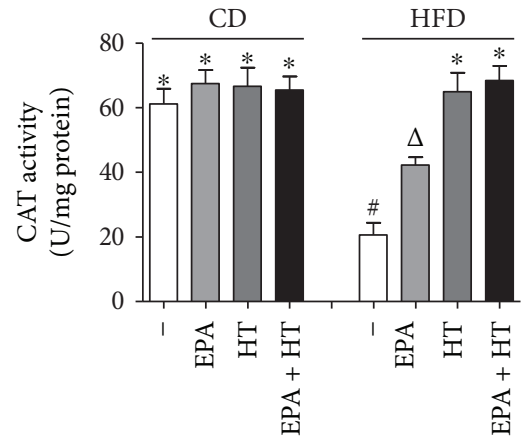

(a)

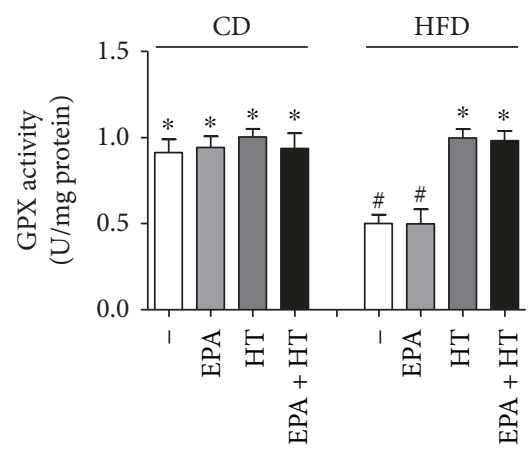

(c)

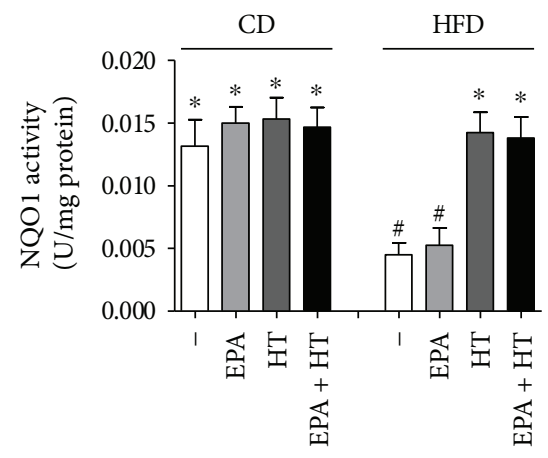

(e)

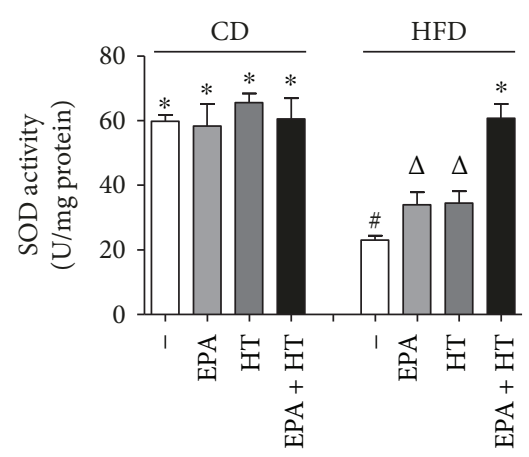

(b)

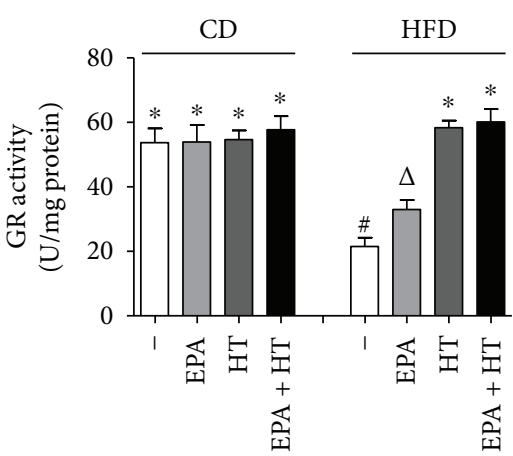

(d)

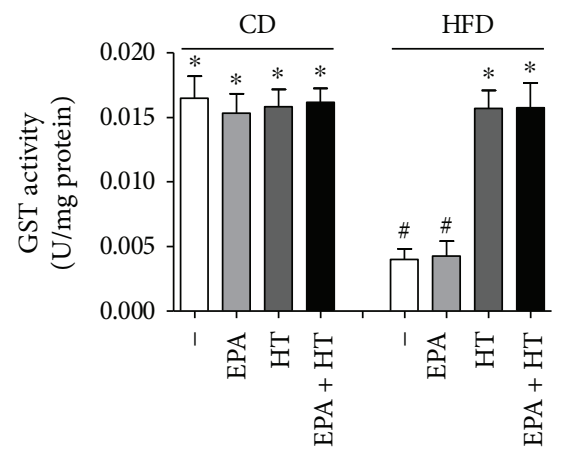

(f)

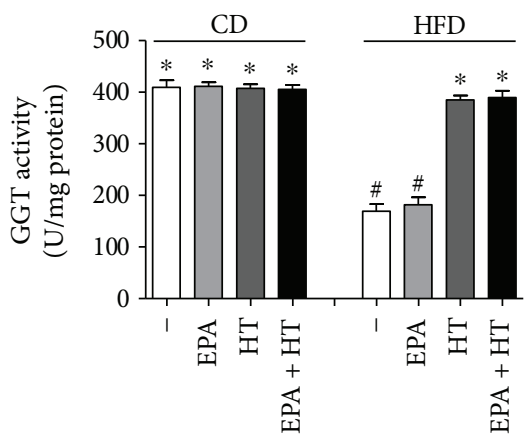

(g)

FIGURE 4: Liver activities of CAT (a), SOD (b), GPX (c), GR (d), NQO1 (e), GST (f), and GGT (g) in mice subjected to control diet (CD) and high-fat diet (HFD) without (-) and with eicosapentaenoic acid (EPA), hydroxytyrosol (HT), and EPA + HT supplementation. Values are means \pm SEM for 8 animals per experimental group. Groups sharing the same symbol are not significantly different among them according to two-way ANOVA and the Bonferroni posttest $(p<0.05)$. CAT: catalase; SOD: superoxide dismutase; GPX: glutathione peroxidase; GR: glutathione reductase; NQO1: NADPH-quinone oxidoreductase 1; GST: glutathione-S-transferase; GGT: $\gamma$-glutamyl transpeptidase. ${ }^{*}$, \# and ${ }^{\Delta}$ indicate the significant differences between the experimental groups. 
regain in n-3 LCPUFA levels, plasma antioxidant capacity, glutathione status, and CAT, GPX, GE, NQO1, GST, and GGT activities, or (ii) showed partial to total recovery transition of Nrf2 functioning and SOD activity, when values for HT alone and EPA + HT are compared. These data indicate that the antisteatotic and antioxidant effects produced by combined EPA + HT supplementation in HFD feeding are mainly due to the HT component, with EPA having a limited contribution at the dosages employed. This conclusion points to antioxidation as a major mechanism underlying attenuation of HFD-induced steatosis by natural products. In agreement with this proposal, mitigation of HFDinduced liver deleterious effects is also attained by both (i) an EVOO type having the highest antioxidant content (859 mg polyphenols/kg; antioxidant capacity of $7156 \mu \mathrm{mol}$ eq. Trolox/L) compared to those having polyphenol levels of 116 or $407 \mathrm{mg} / \mathrm{kg}$ and antioxidant capacities of 3378 or $4841 \mu \mathrm{mol}$ eq. Trolox/L, respectively [34], and (ii) a regular rosa mosqueta oil (RMO) containing $\alpha$ - and $\gamma$-tocopherols compared to a RMO type devoid of tocopherols [44], findings that establish a threshold for the content of antioxidant components of natural products to achieve beneficial effects. In addition to the antioxidant effect of natural products in fatty liver, reduction of HFD-induced liver steatosis by $\mathrm{EPA}, \mathrm{HT}$, and EPA + HT supplementation may result from a direct activation of hepatic lipases such as patatin-like phospholipase domain-containing protein 3 (PNPLA3) which hydrolyses acylglycerols including TGs [45], an aspect that remains to be elucidated in the present model.

\section{Conclusion}

Data presented show that combined EPA + HT supplementation in mice significantly attenuates HFD-induced hepatic steatosis, an effect that mainly relies on HT with a limited contribution of EPA. Under these conditions, the antisteatotic effect of HT is associated with the enhancement in the antioxidant potential of the liver, which partially recovers n-3 LCPUFA levels thus favouring FA oxidation through PPAR- $\alpha$ upregulation [35], while limiting de novo lipogenesis via SREBP-1c downregulation [43]. Additional mechanisms of HT action include (i) prevention of HFD-induced reduction in the desaturation capacity of the liver, with recovery in the activity of $\Delta 5$ and $\Delta 6$ desaturases promoting n-3 LCPUFA repletion [43]; (ii) reduction in the oxidative stress-dependent liver protein carbonylation triggered by HFD, thus decreasing the lipogenic response associated with the endoplasmic reticulum stress (ERS) developed [46, 47], an effect that may be contributed by normalization of SFA levels increased by HFD, FAs that also trigger ERS [48]; and (iii) amelioration of drug-induced cardiotoxicity involving oxidative stress and mitochondrial dysfunction [49], which suggest enhancement in electron transport chain capacity and FA oxidation potential [21]. These considerations and the previous suggestions concerning the adequacy of combination therapies [14-20] reinforce the impact of dietary interventions including safety components addressing oxidative stress as a central mechanism underlying liver steatosis in obesity in particular and other noncommunicable diseases in general.

\section{Data Availability}

The data used to support the findings of this study are available from the corresponding author upon request.

\section{Disclosure}

All authors take responsibility for all aspects of the reliability and freedom from bias of the data presented and their discussed interpretation.

\section{Conflicts of Interest}

The authors declare that there is no conflict of interest regarding the publication of this research article.

\section{Authors' Contributions}

Rodrigo Valenzuela and Luis A. Videla conceived and designed the experiments. Rodrigo Valenzuela, Francisca Echeverría, Andrés Bustamante, Daniela Álvarez, Macarena Ortiz, Sandra A. Soto-Alarcon, Patricio Muñoz, and Alicia Corbari performed the experiments. Rodrigo Valenzuela, Francisca Echeverría, Andrés Bustamante, and Luis A. Videla analysed the data. Rodrigo Valenzuela, Francisca Echeverría, Andrés Bustamante, and Luis A. Videla contributed reagents/ materials/analysis tools. Rodrigo Valenzuela and Luis A. Videla wrote the paper. All authors read and approved the final manuscript.

\section{Acknowledgments}

The authors are grateful to Fondo Nacional de Desarrollo Científico y Tecnológico (National Fund for Scientific and Technological Development, Grant 11140174) granted to Rodrigo Valenzuela, for supporting this study.

\section{References}

[1] H. Sies, "Oxidative stress: a concept in redox biology and medicine," Redox Biology, vol. 4, pp. 180-183, 2015.

[2] L. A. Videla, "Hormetic responses of thyroid hormone calorigenesis in the liver: association with oxidative stress," IUBMB Life, vol. 62, no. 6, pp. 460-466, 2010.

[3] A. K. Singal, S. C. Jampana, and S. A. Weinman, "Antioxidants as therapeutic agents for liver disease," Liver International, vol. 31, no. 10, pp. 1432-1448, 2011.

[4] A. P. Rolo, J. S. Teodoro, and C. M. Palmeira, "Role of oxidative stress in the pathogenesis of non-alcoholic steatohepatitis," Free Radical Biology \& Medicine, vol. 52, no. 1, pp. 59-69, 2012.

[5] L. A. Videla, R. Rodrigo, M. Orellana et al., "Oxidative stressrelated parameters in the liver of nonalcoholic fatty liver disease patients," Clinical Science, vol. 106, no. 3, pp. 261-268, 2004.

[6] S. Seki, T. Kitada, and H. Sakaguchi, "Clinicopathological significance of oxidative cellular damage in non-alcoholic fatty 
liver diseases," Hepatology Research, vol. 33, no. 2, pp. 132134, 2005.

[7] Z. Yesilova, H. Yaman, C. Oktenli et al., "Systemic markers of lipid peroxidation and antioxidants in patients with nonalcoholic fatty liver disease," American Journal of Gastroenterology, vol. 100, no. 4, pp. 850-855, 2005.

[8] R. Valenzuela, A. Espinosa, D. González-Mañán et al., "N-3 long-chain polyunsaturated fatty acid supplementation significantly reduces liver oxidative stress in high fat induced steatosis," PLoS One, vol. 7, no. 10, article e46400, 2012.

[9] R. Valenzuela, C. Barrera, A. Espinosa, P. Llanos, P. Orellana, and L. A. Videla, "Reduction in the desaturation capacity of the liver in mice subjected to high fat diet: relation to LCPUFA depletion in liver and extrahepatic tissues," Prostaglandins, Leukotrienes and Essential Fatty Acids, vol. 98, pp. 7-14, 2015.

[10] G. Tapia, R. Valenzuela, A. Espinosa et al., "N-3 long-chain PUFA supplementation prevents high fat diet induced mouse liver steatosis and inflammation in relation to PPAR- $\alpha$ upregulation and NF- $\kappa$ B DNA binding abrogation," Molecular Nutrition \& Food Research, vol. 58, no. 6, pp. 1333-1341, 2014.

[11] A. R. Soltis, N. J. Kennedy, X. Xin et al., "Hepatic dysfunction caused by consumption of a high-fat diet," Cell Reports, vol. 21, no. 11, pp. 3317-3328, 2017.

[12] J. Willebrords, I. V. A. Pereira, M. Maes et al., "Strategies, models and biomarkers in experimental non-alcoholic fatty liver disease research," Progress in Lipid Research, vol. 59, pp. 106-125, 2015.

[13] J. K. C. Lau, X. Zhang, and J. Yu, "Animal models of nonalcoholic fatty liver disease: current perspectives and recent advances," The Journal of Pathology, vol. 241, no. 1, pp. 3644, 2017

[14] Y. Rotman and A. J. Sanyal, "Current and upcoming pharmacotherapy for non-alcoholic fatty liver disease," Gut, vol. 66, no. 1, pp. 180-190, 2016.

[15] B. C. T. Field, O. B. Chaudhri, and S. R. Bloom, "Obesity treatment: novel peripheral targets," British Journal of Clinical Pharmacology, vol. 68, no. 6, pp. 830-843, 2009.

[16] R. Valenzuela and L. A. Videla, "Crosstalk mechanisms in hepatoprotection: thyroid hormone-docosahexaenoic acid (DHA) and DHA-extra virgin olive oil combined protocols," Pharmacological Research, vol. 132, pp. 168-175, 2018.

[17] M. Mardones, R. Valenzuela, P. Romanque et al., "Prevention of liver ischemia reperfusion injury by a combined thyroid hormone and fish oil protocol," The Journal of Nutritional Biochemistry, vol. 23, no. 9, pp. 1113-1120, 2012.

[18] M. C. Hernández-Rodas, R. Valenzuela, F. Echeverría et al., "Supplementation with docosahexaenoic acid and extra virgin olive oil prevents liver steatosis induced by a high-fat diet in mice through PPAR- $\alpha$ and Nrf2 upregulation with concomitant SREBP-1c and NF- $k$ B downregulation," Molecular Nutrition \& Food Research, vol. 61, no. 12, article 1700479, 2017.

[19] C. Tsioufis and C. Thomopoulos, "Combination drug treatment in hypertension," Pharmacological Research, vol. 125, Part B, pp. 266-271, 2017.

[20] V. G. Athyros, T. K. Alexandrides, H. Bilianou et al., "The use of statins alone, or in combination with pioglitazone and other drugs, for the treatment of non-alcoholic fatty liver disease/non-alcoholic steatohepatitis and related cardiovascular risk. An Expert Panel Statement," Metabolism, vol. 71, pp. 17-32, 2017.
[21] F. Echeverría, M. Ortiz, R. Valenzuela, and L. Videla, "Hydroxytyrosol and cytoprotection: a projection for clinical interventions," International Journal of Molecular Sciences, vol. 18, no. 5, p. 930, 2017.

[22] C. Colica, L. di Renzo, D. Trombetta et al., "Antioxidant effects of a hydroxytyrosol-based pharmaceutical formulation on body composition, metabolic state, and gene expression: a randomized double-blinded, placebo-controlled crossover trial," Oxidative Medicine and Cellular Longevity, vol. 2017, Article ID 2473495, 14 pages, 2017.

[23] R. Valenzuela and L. A. Videla, "The importance of the longchain polyunsaturated fatty acid n-6/n-3 ratio in development of non-alcoholic fatty liver associated with obesity," Food of Function, vol. 2, no. 11, pp. 644-648, 2011.

[24] R. Valenzuela, P. Illesca, F. Echeverría et al., "Molecular adaptations underlying the beneficial effects of hydroxytyrosol in the pathogenic alterations induced by a high-fat diet in mouse liver: PPAR- $\alpha$ and Nrf2 activation, and NF- $\kappa$ B down-regulation," Food \& Function, vol. 8, no. 4, pp. 1526-1537, 2017.

[25] E. G. Bligh and W. J. Dyer, "A rapid method of total lipid extraction and purification," Canadian Journal of Biochemistry and Physiology, vol. 37, no. 1, pp. 911-917, 1959.

[26] I. Rahman, A. Kode, and S. K. Biswas, "Assay for quantitative determination of glutathione and glutathione disulfide levels using enzymatic recycling method," Nature Protocols, vol. 1, no. 6, pp. 3159-3165, 2006.

[27] C. K. Chow, K. Reddy, and A. L. Tappel, "Effect of dietary vitamin $\mathrm{E}$ on the activities of the glutathione peroxidase system in rat tissues," The Journal of Nutrition, vol. 103, no. 4, pp. 618624,1973

[28] H. Lück, "Catalase," in Methods of Enzymatic Assays, H. U. Bergmeyer, Ed., pp. 885-894, Academic Press, New York, 1965.

[29] D. E. Paglia and W. N. Valentine, "Studies on the quantitative and qualitative characterization of erythrocyte glutathione peroxidase," Journal of Laboratory and Clinical Medicine, vol. 70, no. 1, pp. 158-169, 1967.

[30] H. Horn, "Glytathione reductase," in Methods of Enzymatic Assays, H. U. Bergmeyer, Ed., pp. 875-879, Academic Press, New York, 1965.

[31] L. Ernster, F. Navazio, H. Löw, P. Siekevitz, L. Ernster, and E. Diczfalusy, "Soluble diaphorase in animal tissues," Acta Chemica Scandinavica, vol. 12, pp. 595-602, 1958.

[32] W. H. Habig, M. J. Pabst, and W. B. Jakoby, "Glutathione S-transferases. The first enzymatic step in mercapturic acid formation," Journal of Biological Chemistry, vol. 249, no. 22, pp. 7130-7139, 1974.

[33] S. Satomura, Y. Miki, T. Hamanaka, and Y. Sakata, "Kinetic assay of gamma-glutamyltransferase with use of bilirubin oxidase as a coupled enzyme," Clinical Chemistry, vol. 31, no. 8 , pp. 1380-1383, 1985.

[34] M. A. Rincón-Cervera, R. Valenzuela, M. C. Hernandez-Rodas et al., "Supplementation with antioxidant-rich extra virgin olive oil prevents hepatic oxidative stress and reduction of desaturation capacity in mice fed a high-fat diet: effects on fatty acid composition in liver and extrahepatic tissues," Nutrition, vol. 32, no. 11-12, pp. 1254-1267, 2016.

[35] S. Piro, L. Spadaro, M. Russello et al., "Molecular determinants of insulin resistance, cell apoptosis and lipid accumulation in non-alcoholic steatohepatitis," Nutrition, Metabolism \& Cardiovascular Diseases, vol. 18, no. 8, pp. 545-552, 2008. 
[36] J. W. Kaspar, S. K. Niture, and A. K. Jaiswal, "Nrf2:INrf2 (Keap1) signaling in oxidative stress," Free Radical Biology \& Medicine, vol. 47, no. 9, pp. 1304-1309, 2009.

[37] P. Delerive, K. de Bosscher, S. Besnard et al., "Peroxisome proliferator-activated receptor $\alpha$ negatively regulates the vascular inflammatory gene response by negative cross-talk with transcription factors NF- $\kappa \mathrm{B}$ and AP-1," Journal of Biological Chemistry, vol. 274, no. 45, pp. 32048-32054, 1999.

[38] S. Hong, K. Gronert, P. R. Devchand, R. L. Moussignac, and C. N. Serhan, "Novel docosatrienes and 17S-resolvins generated from docosahexaenoic acid in murine brain, human blood, and glial cells. Autacoids and anti-inflammation," Journal of Biological Chemistry, vol. 278, no. 17, pp. 14677-14687, 2003.

[39] B. de Roos, Y. Mavrommatis, and I. A. Brouwer, "Long-chain n-3 polyunsaturated fatty acids: new insights into mechanisms relating to inflammation and coronary heart disease," British Journal of Pharmacology, vol. 158, no. 2, pp. 413-428, 2009.

[40] L. Gao, J. Wang, K. R. Sekhar et al., "Novel n-3 fatty acid oxidation products activate Nrf2 by destabilizing the association between Keap1 and Cullin3," Journal of Biological Chemistry, vol. 282, no. 4, pp. 2529-2537, 2007.

[41] S. Cicerale, L. J. Lucas, and R. S. J. Keast, "Antimicrobial, antioxidant and anti-inflammatory phenolic activities in extra virgin olive oil," Current Opinion in Biotechnology, vol. 23, no. 2, pp. 129-135, 2012.

[42] H. Zrelli, M. Matsuoka, S. Kitazaki et al., "Hydroxytyrosol induces proliferation and cytoprotection against oxidative injury in vascular endothelial cells: role of Nrf2 activation and HO-1 induction," Journal of Agricultural and Food Chemistry, vol. 59, no. 9, pp. 4473-4482, 2011.

[43] R. Valenzuela, F. Echeverria, M. Ortiz et al., "Hydroxytyrosol prevents reduction in liver activity of $\Delta-5$ and $\Delta-6$ desaturases, oxidative stress, and depletion in long chain polyunsaturated fatty acid content in different tissues of high-fat diet fed mice," Lipids in Health and Disease, vol. 16, no. 1, p. 64, 2017.

[44] G. Tapia, D. Silva, N. Romero et al., "Role of dietary $\alpha$ - and $\gamma$-tocopherol from Rosa mosqueta oil in the prevention of alterations induced by high-fat diet in a murine model," Nutrition, vol. 53, pp. 1-8, 2018.

[45] F. V. Bruschi, M. Tardelli, T. Claudel, and M. Trauner, "PNPLA3 expression and its impact on the liver: current perspectives," Hepatic Medicine, vol. 9, pp. 55-66, 2017.

[46] Z. Zheng, C. Zhang, and K. Zhang, "Role of unfolded protein response in lipogenesis," World Journal of Hepatology, vol. 2, no. 6, pp. 203-207, 2010.

[47] H. Malhi and R. J. Kaufman, "Endoplasmic reticulum stress in liver disease," Journal of Hepatology, vol. 54, no. 4, pp. 795809, 2011.

[48] C. L. Gentile, M. A. Frye, and M. J. Pagliassotti, "Fatty acids and the endoplasmic reticulum in non-alcoholic fatty liver disease," BioFactors, vol. 37, no. 1, pp. 8-16, 2011.

[49] S. Granados-Principal, N. el-azem, R. Pamplona et al., "Hydroxytyrosol ameliorates oxidative stress and mitochondrial dysfunction in doxorubicin-induced cardiotoxicity in rats with breast cancer," Biochemical Pharmacology, vol. 90, no. 1, pp. 25-33, 2014. 


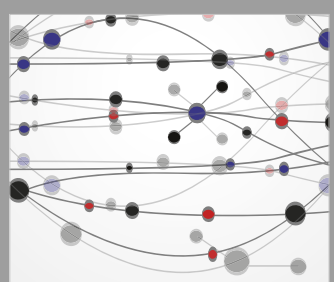

The Scientific World Journal
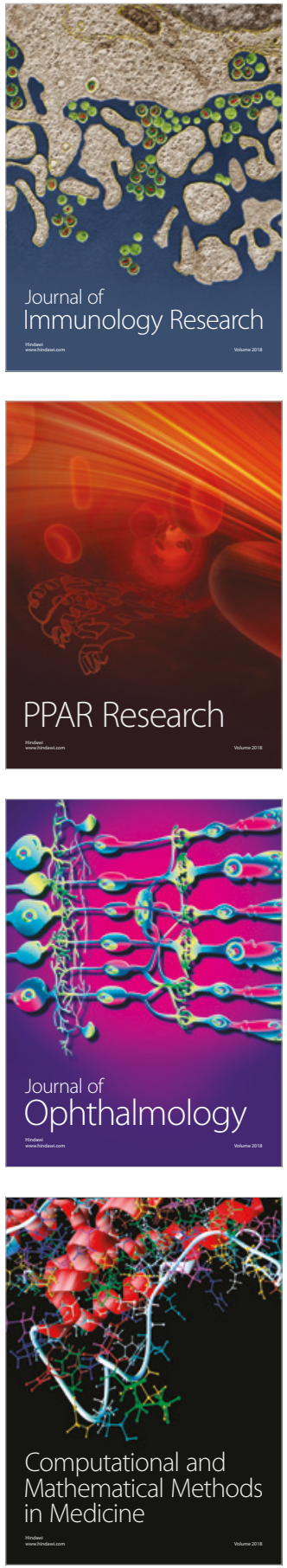

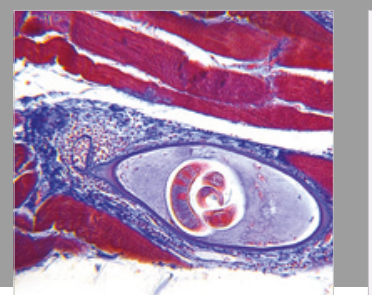

Gastroenterology Research and Practice

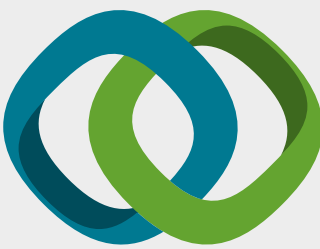

\section{Hindawi}

Submit your manuscripts at

www.hindawi.com
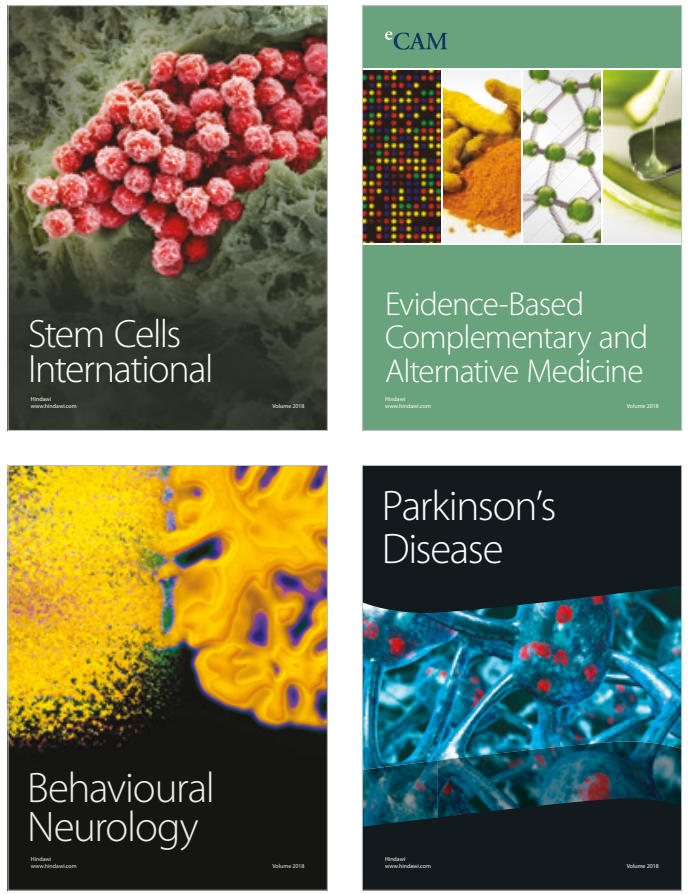

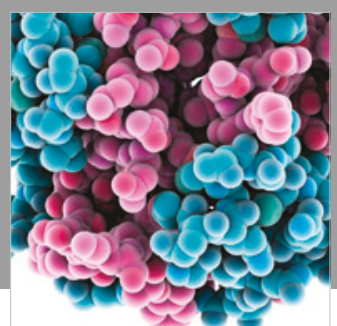

ournal of

Diabetes Research

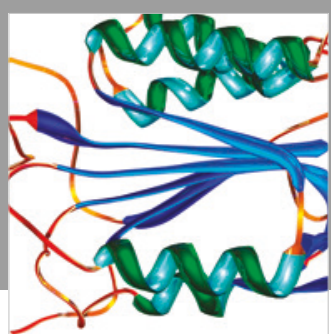

Disease Markers
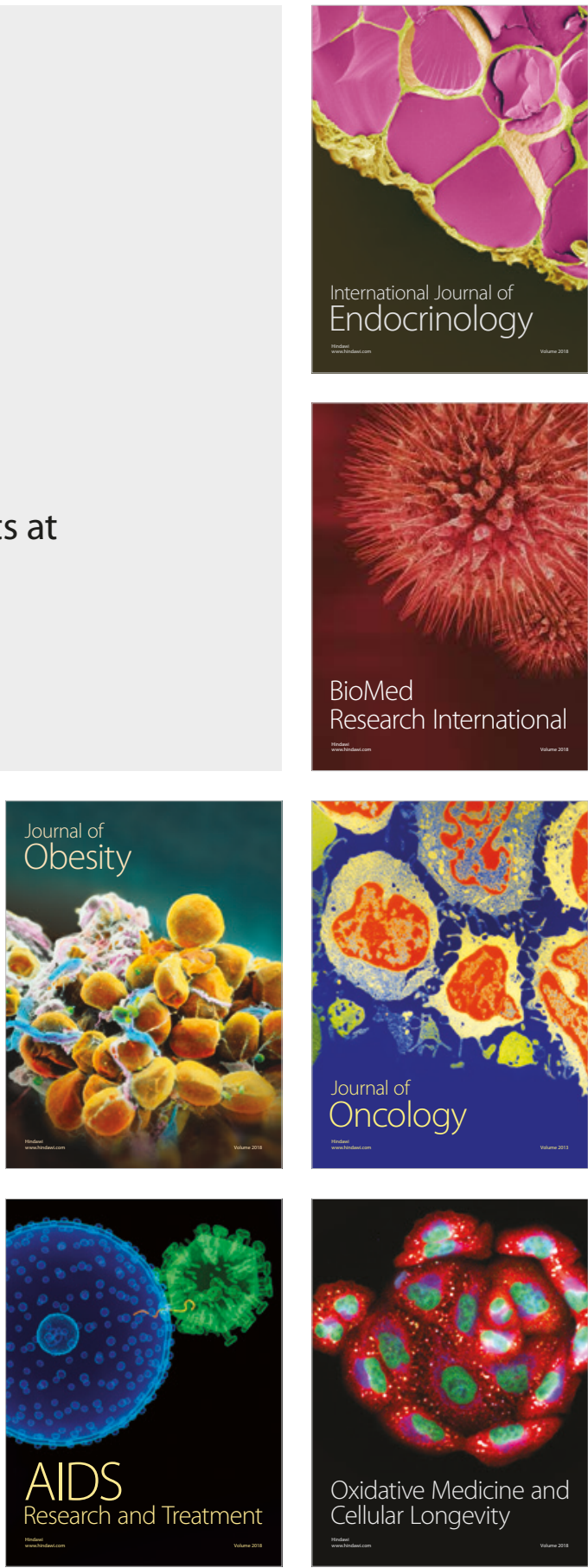